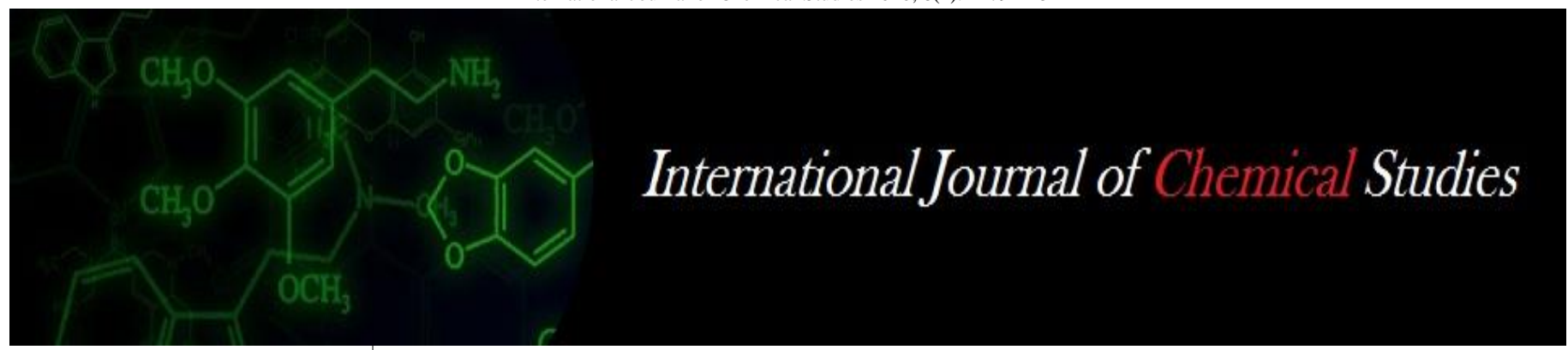

P-ISSN: 2349-8528

E-ISSN: 2321-4902

IJCS 2020; 8(1): 1279-1281

(C) 2020 IJCS

Received: 08-11-2019

Accepted: 12-12-2019

Nivedita Rajput

Department of Agronomy, C S Azad University of Agriculture and Technology, Kanpur, Uttar Pradesh, India

\section{Ram Pyare}

Department of Agronomy, C S Azad University of Agriculture and Technology, Kanpur, Uttar Pradesh, India

\section{Amar Kant Verma}

Department of Soil Conservation and Water Management, C S Azad University of Agriculture and Technology, Kanpur, Uttar Pradesh, India

\section{Pratistha Yadav}

Department of Agronomy, C S Azad University of Agriculture and Technology, Kanpur, Uttar Pradesh, India

\section{Rahul Ranjan}

Department of Soil Science and Agricultural Chemistry, C S Azad University of Agriculture and Technology, Kanpur, Uttar Pradesh, India

\section{Vijay Kumar Rajpoot}

Department of Soil Conservation and Water Management, C S Azad University of Agriculture and Technology, Kanpur, Uttar Pradesh, India

Corresponding Author: Amar Kant Verma Department of Soil Conservation and Water Management, C S Azad University of Agriculture and Technology, Kanpur, Uttar Pradesh, India

\section{Evaluation of the effect of Acadian soil health granule (SoliGro Gr) on Growth and yield of paddy (Oryza sativa L.)}

\author{
Nivedita Rajput, Ram Pyare, Amar Kant Verma, Pratistha Yadav, Rahul \\ Ranjan and Vijay Kumar Rajpoot
}

DOI: https://doi.org/10.22271/chemi.2020.v8.i1r.8432

\begin{abstract}
An experiment was conducted at the Student's Instructional Farm of Chandra Sekhar Azad University of Agriculture and Technology, Kanpur during kharif season of 2017 to find out the performance of biostimulant (SoliGro Gr) on growth and yield of paddy and also their suitable application. The experiment comprises of ten treatments. Laid out in Randomized Block Design with three replications. The soil of the experimental field was sandy loam in texture with $\mathrm{PH} 7.6$ and $\mathrm{EC}$ of $0.37 \mathrm{~mm}$ hos $\mathrm{m}^{-1}$ at $25^{\circ} \mathrm{C}$. It contained $0.4 \%$ organic carbon, $183.0 \mathrm{~kg}$ available $\mathrm{N} / \mathrm{ha}, 18.0 \mathrm{~kg}$ available phosphorous $/ \mathrm{ha}$ and 235 $\mathrm{kg} / \mathrm{ha}$ available potassium. The recommended dose of fertilizes (NPK:120:60:60 kg/ha) were applied uniformly in each plot. The soil Health Granule was applied at final land preparation before transplanting, 25, $45 \& 65$ DAT in rice crop as per treatments. Result showed that the application of RDF+SoliGro @ $10 \mathrm{~kg} / \mathrm{ha}$ at FLP \& 25 DAT promoted significantly the growth and yield attributes of rice crop viz. plant stand count at initial stage and also at maturity as compared to control treatment, plant height at all stages were, effective tillers/hill, number of grains/panicle, effective tillers $/ \mathrm{m}^{2}$, root hairs/hill, root length, root dry weight/hill at different stages were observed significantly as compared to control treatment. Application of RDF+SoliGro @ $10 \mathrm{~kg} / \mathrm{ha}$ at FLP \& 25 DAT recorded significantly higher Soil Microbial Population (cfu/ml) at PDA (106 \& 107 dilution) and NA (108 \& 109 dilution), respectively compared to control treatment. Application of RDF+SoliGro @ $10 \mathrm{~kg} / \mathrm{ha}$ at FLP \& 25 DAT also recorded significantly maximum grain yield $(53.46 \mathrm{q} / \mathrm{ha})$ and harvest index $(37.30 \%)$ compared to all rest treatment. The net income was increased in application of RDF with two times used SoliGro Gr @ 10 $\mathrm{kg} / \mathrm{ha}$ at final land preparation and 25 days after transplanting of rice by a margin of $64.32 \%, 28.04 \%$, $4.59 \%, 30.19 \%, 37.84 \%, 16.42 \%, 23.86 \%, 7.26 \%$ and $10.27 \%$ in respect of net return over remaining treatments, respectively.
\end{abstract}

Keywords: Seed yield, soil health granule and economics

\section{Introduction}

Rice is an important kharif cereal (food grain) crop of not only in India but world as a whole. It occupies $23.3 \%$ of gross cropped area of the country. Rice contributes $43 \%$ of total food grain production and $46 \%$ of total cereal production of the country. In India it is mainly concern to rice which is the staple food of majority of our population like most of the people of South East Asia. It is the principle food of 50 percent of the human global population and 90 percent of India's population. India rank first among all the rice producing countries of the world in respect to area which is about 43.20 million hectares with annual production of about 89.13 million tones (2009-10) which is less than previous year 99.18 million tone (2008-09) and ranks second in production next to china. Among other countries Indonesia, Malaysia, Bangladesh, Pakistan, Burma, Thailand, Japan, and Philippines are important in regards rice cultivation. The productivity is higher than Thailand, Pakistan, Bangladesh and Nepal but much below the productivity in Japan, Korea, USA and Indonesia. The world wide global consumption of rice is about $\mathbf{4 7 5 . 6 5}$ million tones. China is leading rice consumer that year about 141.45 million tone (Anonymous, 2018) ${ }^{[1]}$. The various top ten rice producing countries globally are listed here with sequence as China ranks first and it produced about 202.6 million metric ton, India produced 155.7 million metric ton, Indonesia produced 65.7 million metric ton, Bangladesh produced 50.6 million metric ton, Vietnam produced 42.3 million metric ton, 
Thailand produced 34.5 million metric ton, Burma produced 32.8 million metric ton, Philippines produced 16.6 million metric ton, Brazil produced 13.5 million metric ton and Pakistan produced of about 9.2 million metric ton. The production details are given by the source of Food and Agriculture Organization with data of 2017 (Kumar et al, 2017) ${ }^{[6]}$.

Seaweed extracts (Ascophyllum nodosum) extract based organic granular product for soil application. SoliGro GR is a soil health product powered by Acadian Bio Switch bioactive compound which enhances natural processes within soil resulting in root development, microbial activity, soil conditioning, plant growth and help protect crops against environmental stresses. It is a soil health product containing an exclusive mixture of beneficial bioactive compounds such as polysaccharides, organic acids that invigorate soil environment particularly by promoting the activities of beneficial soil micro-organism. SoliGro GR provides balanced nutrition for optimum growth, yield and quality, it helps plants to overcome abiotic environmental stresses like drought, salinity, cold and frost efficiency and finally, boosts the immune system of the plants.

\section{Materials and Methods}

The experiment was conducted during rabi season of 2017-18 in student's Instructional Farm (SIF) of C S Azad University of Agriculture \& Technology, Kanpur in alluvial soil. Soil of the experimental plot was sandy loam in texture and slightly calcareous having organic carbon $0.33 \%$, total nitrogen $0.034 \%$, available $\mathrm{P}_{2} \mathrm{O}_{5} 16.8 \mathrm{ha}^{-1}, \mathrm{pH} 7.6$, electrical conductivity $0.38 \mathrm{dSm}^{-1}$, permanent wilting point $6.3 \%$, field capacity $18.3 \%$, maximum water holding capacity $29.8 \%$, Bulk density $1.45 \mathrm{Mgm}^{-3}$, particle density $2.57 \mathrm{Mgm}^{-3}$ and porosity $43.5 \%$. The experiment was conducted in a randomized block design with three replications and 10 treatments viz. Control (RDF), RDF + SoliGro Gr @ $10 \mathrm{~kg} / \mathrm{ha}$ at FLP, RDF+SoliGro @ $10 \mathrm{~kg} / \mathrm{ha}$ at 25 DAT, RDF+SoliGro @ $10 \mathrm{~kg} / \mathrm{ha}$ at $45 \mathrm{DAT}, \mathrm{RDF}+$ SoliGro @ $10 \mathrm{~kg} / \mathrm{ha}$ at 65 DAT, RDF+SoliGro @ $10 \mathrm{~kg} / \mathrm{ha}$ at FLP \& $25 \mathrm{DAT}$, RDF+SoliGro@ 10 kg/ha at FLP \& 45 DAT, RDF +SoliGro @ $10 \mathrm{~kg} / \mathrm{ha}$ at FLP \& 65 DAT, RDF+SoliGro @ $10 \mathrm{~kg} / \mathrm{ha}$ at FLP, 25 \& 45 DAT, RDF+SoliGro @ 10 kg/ha at FLP, 25, 45 \& 65 DAT. The paddy variety NDR-359 was transplanted on dated 29, July 2017 at spacing of $20 \mathrm{~cm} \times 10 \mathrm{~cm}$ and crop harvested on dated 04, November 2017. Full dose of P and K while half dose of $\mathrm{N}$ was applied as basal dose at the time of sowing where rest of $\mathrm{N}$ was given in two split doses during experimentation. Available moisture at sowing time upto 100 $\mathrm{cm}$ soil profile was $277.2 \mathrm{~mm}$. Whereas amount of rainfall received during the crop period was nil against the average annual rainfall of about $800 \mathrm{~mm}$. Recommended package of practices were applied in different treatments. Soil moisture was monitored gravimetrically using the sample collected from 0-25, 25-50, 50-75 and 75-100 cm soil depths at regular monthly intervals to quantify the soil moisture content and growth parameters by randomly selecting three plants for each plots till the harvest.

The data collected on growth, yield attributes and yields were statistically analyzed (Fisher and Yates, 1958). Recommended package of practices and fertilizers doses were applied in different treatments. The harvest index was worked out with the help of following formula:

$$
\text { Harvest Index }(\%)=\frac{\text { Seed yield }\left(\mathrm{q} \mathrm{ha}^{-1}\right)}{\text { Biological yield }\left(\mathrm{q} \mathrm{ha}^{-1}\right)} \times 100
$$

For economic evaluation the cost of cultivation, gross returns, net returns, and $\mathrm{B}: \mathrm{C}$ ratio were computed using standard procedure based on minimum support price of Indian mustard. Root studies were made at harvest by selecting two plants at random from each plot. The roots were freed with a fine jet of water spray so that the delicate rootlets were not broken.

\section{Results and Discussion}

The application of seaweed extracts (SoliGro Gr @ $10 \mathrm{~kg} / \mathrm{ha}$ ) at final land preparation time and 25 days after transplanting of crop with recommended dose of fertilizers promoting growth attributes viz., plant height, tillers/hill, functional leaves/plant and yield parameters viz., 67 panicle length, total grain/panicle, effective tillers $\left(\mathrm{m}^{2}\right)$ and test weight of NDR359 variety of rice crop. Similar observation has also been recorded by Aroubandi, (2017) ${ }^{[2]}$.

Two times applied seaweed extracts as SoliGro Gr @ 10 $\mathrm{kg} / \mathrm{ha}$ at final land preparation time and 25 days after transplanting of rice crop along with RDF enhanced root development viz. the root hairs/hill, maximum root length at different days stages and root dry weight at 30, 60 and 90 days stage and soil microbial population viz. PDA (106 and 107 dilution) and NA (108 and 109 dilution) during experimental year of study. Similar observation has also been recorded by Jisan et al., (2014) ${ }^{[4]}$.

The rice yields of biological, grain and straw yield were recorded in recommended dose of fertilizer and applied two time SoliGro Gr @ $10 \mathrm{~kg} / \mathrm{ha}$ at final land preparation time and 25 days after transplanting of crop in present investigation. Further, the treatment of recommended dose of fertilizer along with two times applied SoliGro Gr @ $10 \mathrm{~kg} / \mathrm{ha}$ at final land preparation time and 25 days after transplanting of rice crop statistically increased the Gross income, net income and return per rupee investment in present field experimentation. Similar observation has also been recorded by Kavitha et al., (2008) ${ }^{[5]}$ and Verma and Yadav, (2018) ${ }^{[7]}$.

Table 1: Effect on Plant stand, Plant Height, Yield attributes under different treatments

\begin{tabular}{|c|c|c|c|c|c|c|c|c|c|c|c|c|}
\hline \multirow{2}{*}{ Treatments } & \multicolumn{2}{|c|}{ Plant population $\left(000 \mathrm{ha}^{-1}\right)$} & \multicolumn{4}{|c|}{ Plant Height (cm) } & \multirow{2}{*}{$\begin{array}{c}\text { Effective } \\
\text { Tiller hill- }_{1}^{-}\end{array}$} & \multirow{2}{*}{$\begin{array}{c}\text { Functiona } \\
1 \text { leaves } \\
\text { plant }^{-1}\end{array}$} & \multirow{2}{*}{\begin{tabular}{|c|} 
Panicle \\
length \\
(cm)
\end{tabular}} & \multirow{2}{*}{\begin{tabular}{|c|} 
Total \\
grain \\
panicle $^{-1}$
\end{tabular}} & \multirow{2}{*}{\begin{tabular}{|l|} 
Effective \\
tiller $\mathbf{~ m}^{-2}$
\end{tabular}} & \multirow{2}{*}{$\begin{array}{c}\text { Test } \\
\text { weigh } \\
t(g)\end{array}$} \\
\hline & Initial & Final & 30 DAS & 60 DAS & 90 DAS & At Harvest & & & & & & \\
\hline $\mathrm{T}_{1}$ & 238.45 & 235.35 & 16.25 & 40.56 & 86.72 & 90.45 & 7.88 & 15.88 & 20.55 & 123.40 & 376.12 & 22.47 \\
\hline $\mathrm{T}_{2}$ & 243.65 & 240.16 & 18.55 & 43.88 & 88.80 & 91.34 & 8.10 & 16.67 & 20.62 & 131.16 & 398.80 & 23.19 \\
\hline $\mathrm{T}_{3}$ & 279.67 & 276.39 & 22.01 & 48.23 & 91.44 & 92.78 & 8.77 & 17.66 & 20.71 & 139.73 & 430.50 & 24.20 \\
\hline $\mathrm{T}_{4}$ & 254.62 & 252.46 & 18.49 & 42.77 & 88.64 & 91.23 & 7.99 & 16.20 & 20.60 & 130.20 & 389.50 & 23.15 \\
\hline $\mathrm{T}_{5}$ & 278.47 & 274.26 & 17.34 & 41.66 & 87.64 & 89.00 & 7.99 & 16.09 & 20.58 & 128.30 & 380.30 & 23.07 \\
\hline $\mathrm{T}_{6}$ & 280.46 & 278.35 & 23.06 & 51.11 & 93.67 & 94.56 & 8.77 & 17.66 & 20.75 & 140.40 & 438.50 & 24.98 \\
\hline $\mathrm{T}_{7}$ & 240.12 & 237.10 & 19.44 & 45.50 & 90.25 & 91.34 & 8.55 & 16.99 & 20.64 & 132.60 & 426.05 & 23.85 \\
\hline $\mathrm{T}_{8}$ & 265.37 & 261.24 & 19.24 & 44.49 & 89.97 & 90.56 & 8.22 & 16.77 & 20.62 & 131.40 & 409.20 & 23.25 \\
\hline
\end{tabular}




\begin{tabular}{|c|c|c|c|c|c|c|c|c|c|c|c|c|}
\hline $\mathrm{T}_{9}$ & 283.67 & 279.67 & 19.77 & 46.11 & 90.83 & 92.54 & 8.66 & 17.52 & 20.65 & 134.40 & 428.34 & 24.25 \\
\hline $\mathrm{T}_{10}$ & 285.34 & 282.15 & 20.79 & 46.66 & 91.23 & 93.67 & 8.66 & 17.55 & 20.67 & 137.30 & 430.10 & 24.35 \\
\hline SE (d) & 0.356 & 0.401 & 1.472 & 2.348 & 1.658 & 1.76 & 0.280 & 0.477 & 0.112 & 4.104 & 6.031 & 0.779 \\
\hline $\mathrm{CD}(\mathrm{P}=0.05)$ & 0.721 & 0.865 & 3.092 & 4.933 & 3.433 & 3.561 & 0.589 & 1.003 & N.S. & 8.622 & 12.967 & 1.69 \\
\hline
\end{tabular}

T1: Control (RDF), T2: RDF + SoliGro Gr @ $10 \mathrm{~kg} / \mathrm{ha}$ at FLP, T3: RDF+SoliGro @ $10 \mathrm{~kg} / \mathrm{ha}$ at $25 \mathrm{DAT}$, T4: RDF+SoliGro @ $10 \mathrm{~kg} / \mathrm{ha}$ at 45 DAT, T5: RDF+SoliGro @ $10 \mathrm{~kg} / \mathrm{ha}$ at 65 DAT, T6: RDF+SoliGro @ $10 \mathrm{~kg} / \mathrm{ha}$ at FLP \& 25 DAT, T7: RDF+SoliGro@ 10 kg/ha at FLP \& 45 DAT, T8: RDF +SoliGro @ $10 \mathrm{~kg} / \mathrm{ha}$ at FLP \& 65 DAT, T9: RDF+SoliGro @ $10 \mathrm{~kg} / \mathrm{ha}$ at FLP and T10: 25 \& 45 DAT, RDF+SoliGro @ $10 \mathrm{~kg} / \mathrm{ha}$ at FLP, 25, 45 \& 65 DAT.

Table 2: Effect on Root study and Soil microbial population $(\mathrm{cfe} / \mathrm{ml})$ under different treatments

\begin{tabular}{|c|c|c|c|c|c|c|c|c|c|c|c|}
\hline \multirow[b]{2}{*}{ Treatments } & \multirow{2}{*}{$\begin{array}{c}\text { Root } \\
\text { hairs/hills }\end{array}$} & \multicolumn{3}{|c|}{ Maximum root length $(\mathrm{cm})$} & \multicolumn{3}{|c|}{ Root dry weight $\left(\mathrm{g}\right.$ hill- $\left.{ }^{-1}\right)$} & \multicolumn{4}{|c|}{ Soil microbial population $(\mathrm{cfe} / \mathrm{ml})$} \\
\hline & & 30 days & 60 days & 90 days & 30 days & 60 days & 90 days & $\begin{array}{l}\text { PDA }\left(1^{6}\right. \\
\text { dilution) }\end{array}$ & $\begin{array}{l}\text { PDA (10 }{ }^{7} \\
\text { dilution) }\end{array}$ & $\begin{array}{c}\text { PDA }\left(10^{8}\right. \\
\text { dilution) }\end{array}$ & $\begin{array}{c}\text { PDA (109 } \\
\text { dilution) }\end{array}$ \\
\hline $\mathrm{T}_{1}$ & 60.00 & 9.56 & 10.33 & 11.07 & 4.36 & 4.90 & 5.37 & 1.66 & 1.33 & 42.00 & 40.66 \\
\hline $\mathrm{T}_{2}$ & 66.66 & 10.00 & 10.78 & 11.37 & 4.59 & 5.12 & 5.54 & 3.66 & 2.33 & 68.33 & 44.66 \\
\hline $\mathrm{T}_{3}$ & 75.99 & 10.22 & 11.32 & 11.95 & 4.44 & 5.33 & 5.87 & 15.00 & 5.66 & 91.66 & 66.33 \\
\hline $\mathrm{T}_{4}$ & 66.11 & 9.60 & 10.59 & 11.26 & 4.34 & 4.97 & 5.51 & 2.33 & 2.00 & 63.00 & 43.33 \\
\hline $\mathrm{T}_{5}$ & 60.22 & 9.55 & 10.39 & 11.01 & 4.37 & 4.91 & 5.49 & 1.66 & 1.66 & 44.33 & 41.66 \\
\hline $\mathrm{T}_{6}$ & 76.66 & 11.05 & 11.37 & 11.96 & 4.50 & 5.46 & 5.99 & 15.33 & 7.00 & 92.33 & 70.66 \\
\hline $\mathrm{T}_{7}$ & 67.77 & 10.55 & 11.16 & 11.76 & 4.55 & 5.17 & 5.67 & 4.66 & 5.00 & 70.66 & 58.33 \\
\hline $\mathrm{T}_{8}$ & 66.78 & 10.53 & 10.79 & 11.14 & 4.52 & 5.12 & 5.60 & 4.33 & 2.66 & 68.33 & 49.66 \\
\hline $\mathrm{T}_{9}$ & 70.11 & 10.66 & 11.24 & 11.87 & 4.57 & 5.21 & 5.68 & 7.00 & 5.00 & 77.00 & 60.66 \\
\hline $\mathrm{T}_{10}$ & 72.77 & 10.66 & 11.31 & 11.95 & 4.56 & 5.28 & 5.78 & 8.00 & 5.33 & 81.00 & 61.66 \\
\hline SE (d) & 3.600 & 0.457 & 0.239 & 0.257 & 0.123 & 0.126 & 0.144 & 0.910 & 0.595 & 6.142 & 4.292 \\
\hline $\mathrm{CD}(\mathrm{P}=0.05)$ & 7.564 & 0.961 & 0.503 & 0.540 & 0.282 & 0.266 & 0.302 & 1.899 & 1.241 & 12.818 & 8.956 \\
\hline
\end{tabular}

$\mathrm{T}_{1:}$ Control (RDF), T2: RDF + SoliGro Gr @ $10 \mathrm{~kg} / \mathrm{ha}$ at FLP, T3: RDF+SoliGro @ $10 \mathrm{~kg} / \mathrm{ha}$ at $25 \mathrm{DAT}$, T4: RDF+SoliGro @ $10 \mathrm{~kg} / \mathrm{ha}$ at 45 DAT, T5: RDF+SoliGro @ $10 \mathrm{~kg} / \mathrm{ha}$ at 65 DAT, T6: RDF+SoliGro @ $10 \mathrm{~kg} / \mathrm{ha}$ at FLP \& 25 DAT, T7: RDF+SoliGro@ 10 kg/ha at FLP \& 45 DAT, T8: RDF +SoliGro @ $10 \mathrm{~kg} / \mathrm{ha}$ at FLP \& 65 DAT, T9: RDF+SoliGro @ $10 \mathrm{~kg} / \mathrm{ha}$ at FLP and T10: 25 \& 45 DAT, RDF+SoliGro @ $10 \mathrm{~kg} / \mathrm{ha}$ at FLP, 25, 45 \& 65 DAT.

Table 3: Effect on Biological yield, Grain yield, Straw yield, Harvest index, and Economics under different treatments

\begin{tabular}{|c|c|c|c|c|c|c|c|c|}
\hline Treatments & $\begin{array}{c}\text { Biological yield } \\
\left(\mathrm{q} \mathrm{ha}^{-1}\right)\end{array}$ & \begin{tabular}{|c|}
$\begin{array}{c}\text { Grain yield } \\
\left(\mathrm{q} \mathbf{h a}^{-1}\right)\end{array}$ \\
\end{tabular} & \begin{tabular}{|c} 
Straw yield \\
$\left(\mathbf{q}\right.$ ha $\left.^{-1}\right)$
\end{tabular} & \begin{tabular}{|c|} 
Harvest index \\
$(\%)$
\end{tabular} & \begin{tabular}{|c|}
$\begin{array}{c}\text { Cost of cultivation } \\
(\text { Rs. ha-1) }\end{array}$ \\
\end{tabular} & \begin{tabular}{|c|}
$\begin{array}{c}\text { Gross income } \\
(\text { Rs. ha }\end{array}$ he $\left.^{-1}\right)$ \\
\end{tabular} & \begin{tabular}{|c|}
$\begin{array}{c}\text { Net income } \\
\left(\text { Rs. ha }^{-1}\right)\end{array}$ \\
\end{tabular} & \begin{tabular}{|c|}
$\begin{array}{c}\text { Return Rupees }^{-1} \\
(\text { Rs. ha' }\end{array}$ \\
\end{tabular} \\
\hline $\mathrm{T}_{1}$ & 124.13 & 41.53 & 75.58 & 33.45 & 51000 & 77826 & 26826 & 0.53 \\
\hline $\mathrm{T}_{2}$ & 131.73 & 46.55 & 83.18 & 35.33 & 52500 & 86927 & 34427 & 0.67 \\
\hline $\mathrm{T}_{3}$ & 141.42 & 51.02 & 88.02 & 36.08 & 52500 & 94644 & 42144 & 0.80 \\
\hline $\mathrm{T}_{4}$ & 131.73 & 46.30 & 82.23 & 35.42 & 52500 & 86359 & 33859 & 0.64 \\
\hline $\mathrm{T}_{5}$ & 129.13 & 45.33 & 80.16 & 35.10 & 52500 & 84480 & 31980 & 0.61 \\
\hline $\mathrm{T}_{6}$ & 143.31 & 53.46 & 86.78 & 37.30 & 54000 & 98081 & 44081 & 0.82 \\
\hline $\mathrm{T}_{7}$ & 139.07 & 49.42 & 86.20 & 35.54 & 54000 & 91864 & 37864 & 0.70 \\
\hline $\mathrm{T}_{8}$ & 136.78 & 48.04 & 85.25 & 35.12 & 54000 & 89590 & 35590 & 0.66 \\
\hline $\mathrm{T}_{9}$ & 144.52 & 52.14 & 89.32 & 36.07 & 55500 & 96595 & 41095 & 0.74 \\
\hline $\mathrm{T}_{10}$ & 144.82 & 52.29 & 90.09 & 36.11 & 57000 & 96976 & 39976 & 0.70 \\
\hline SE (d) & 3.878 & 2.805 & 3.455 & 1.332 & - & - & - & - \\
\hline $\mathrm{CD}(\mathrm{P}=0.05)$ & 8.150 & 7.098 & 7.262 & 2.800 & - & - & - & - \\
\hline
\end{tabular}

$\mathrm{T}_{1:}$ Control (RDF), T2: RDF + SoliGro Gr @ $10 \mathrm{~kg} / \mathrm{ha}$ at FLP, T3: RDF+SoliGro @ $10 \mathrm{~kg} / \mathrm{ha}$ at $25 \mathrm{DAT}, \mathrm{T}$ : RDF+SoliGro @ $10 \mathrm{~kg} / \mathrm{ha}$ at 45 DAT, T5: RDF+SoliGro@ $10 \mathrm{~kg} / \mathrm{ha}$ at 65 DAT, T6: RDF+SoliGro @ $10 \mathrm{~kg} / \mathrm{ha}$ at FLP \& 25 DAT, T7: RDF+SoliGro@ $10 \mathrm{~kg} / \mathrm{ha}$ at FLP \& 45 DAT, T8: RDF +SoliGro @ $10 \mathrm{~kg} / \mathrm{ha}$ at FLP \& 65 DAT, T9: RDF+SoliGro @ $10 \mathrm{~kg} / \mathrm{ha}$ at FLP and T10: 25 \& 45 DAT, RDF+SoliGro @ $10 \mathrm{~kg} / \mathrm{ha}$ at FLP, 25, 45 \& 65 DAT.

\section{Conclusion}

On the basis of the conclusions derived from present study, a treatment of recommended dose of fertilizer with two times applied SoliGro Gr @ 10 kg/ha at final land preparation time and 25 days after transplanting may be recommended for NDR-359 variety of rice in condition of central zone of Uttar Pradesh.

\section{References}

1. Anonymous. The Hindu survey of Indian agriculture, 2018, 21-24.

2. Aroubandi $H$. Impact of silicon in managing rice diseases, Yield and quality of rice. Scinzer J Agric. Biol. Sci, 2017; 3(1):22-36.

3. Fichers RA, Yates YE. Report on coordination of fishers statistics in India. A. Handbook of Agricultural statistics. 1958; $17: 47$.
4. Jisan MT, Paul SK, Salim M. Yield performance of some transplant Aman rice varieties as influenced by different levels of nitrogen. Journal of the Bangladesh Agricultural University. 2014; 12(2):321-324.

5. Kavitha V, Ganesaraja, Paulpandi VK. Effect of foliar application of seaweed extract on growth and yield of rice (Oryza sativa L.) Agric. Sci. Digest. 2008; 28(2):127-129.

6. Kumar SS, Singh RK, Yadav MN. Production potential and returns of Musterd intercropping systems under rainfed conditions of Haryana. Indian Journal of Environmental Sciences. 2017; 32(1):345-350.

7. Verma, Amar Kant, Yadav PN. Productivity and wateruse efficiency of linseed (Linum usitatissimum L.)-based cropping systems as influenced by integrated nutrient management under rainfed condition. Journal of Soil and Water Conservation. 2018; 17(1):53-57. 